\title{
NONEXISTENCE OF SOLUTION FOR HIGHER ORDER EVOLUTION EQUATIONS AND INEQUALITIES*
}

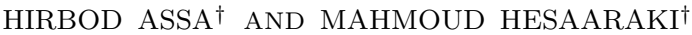

\begin{abstract}
Many authors have established results for existence and nonexistence of nonlinear evolution P.D.Es in the form $\frac{\partial^{k} u}{\partial t^{k}}-\delta_{1} \Delta u+\delta_{2} \Delta^{2} u=f(x, \nabla u, u)$ where $\delta_{1}, \delta_{2} \in\{0,1\}$. In this paper we will prove the nonexistence results for the general form that contains this forms for $f(x, \nabla u, u)=$ $|x|^{-\gamma}|u|^{q}$ or $|u|^{q}$ or $\left|\nabla u^{q}\right|^{p}$.
\end{abstract}

Key words. nonexistence of solution, nonglobal solution

AMS subject classifications. 35L15, 35L70, 35A70

1. Introduction. Consider the following family of inequalities in a domain $\Omega \subseteq$ $\mathbb{R}^{N}$,

$$
\sum_{i=1}^{k} \frac{\partial^{i}}{\partial t^{i}}\left(a_{i}(t, x) u(t, x)\right)+\sum_{|\alpha| \leq n} D^{\alpha}\left(b_{\alpha}(t, x) u^{m_{\alpha}}(t, x)\right) \geq c(t, x) F(u(t, x))
$$

where $F(u)=|x|^{-\gamma}|u|^{q}$ or $|u|^{q}$ or $\left|\nabla u^{q}\right|^{p}$ for $\gamma>0, p, q>1, m_{\alpha}>0$ are real numbers. Here $a_{i}(t, x), b_{\alpha}(t, x)$ and $c(t, x)$ are real valued functions and $D^{\alpha}=\frac{\partial^{\alpha_{1}}}{\partial x_{1} \alpha_{1}} \ldots \frac{\partial^{\alpha_{N}}}{\partial x_{N} \alpha_{N}}$. In the present paper we will to investigate nonexistence of solution in bounded domain for finite interval of time and nonexistence of global solution in $\mathbb{R}^{N}$ with suitable initial data on $[0,+\infty)$ and as a result of these, nonexistence of nonnegative solution for nonevolution P.D.Es.

Suppose that $u(0, x)=u_{0}(x), \ldots, \frac{\partial^{k-1}}{\partial t^{k-1}} u(0, x)=u_{k-1}(x)$ are initial data for (1.1). By initial data in this paper we mean:

$$
\frac{\partial^{i-j}}{\partial t^{i-j}}\left(a_{i}(0, x) u(0, x)\right):=\sum_{l_{1}+l_{2}=i-j} \frac{(i-j) !}{l_{1} ! l_{2} !} \frac{\partial^{l_{1}}}{\partial t^{l_{1}}} a_{i}(0, x) u_{l_{2}}(x)
$$

for all $1 \leq j \leq i \leq k$, when $a_{i}(t, x)$ is considered to be $i-1$ time $t$-differentiable at $t=0(0 \leq i \leq k-1)$.

For $F(u)=|u|^{q}$, we shall prove the nonexistence of nontrivial global solution if the value of initial data are large. In this case our result is independent on boundary condition. For $F(u)=\left|\nabla u^{q}\right|^{p}$ we will consider of the nonexistence of nonnegative nontrivial global solution for the Dirichlet boundary condition when $p-1>n+1$ and large initial data.

Now consider the following problem:

\footnotetext{
*Received February 4, 2004; accepted for publication January 19, 2005.

$\dagger$ Department of Mathematics, Sharif University of Technology, P.O. Box 11365-9415, Tehran, Iran (asa@mehr.sharif.edu; hesaraki@sina.sharif.ac.ir). Current address of the first author: Département de Mathématiques et de Statistique, Université de Montréal, CP 6128 succ Centre-Ville, Montréal QC H3C 3J7, Canada (assa@dms.umontreal.ca).
} 


$$
\left\{\begin{array}{l}
\sum_{i=1}^{k} \frac{\partial^{i}}{\partial t^{i}}\left(a_{i}(t, x) u(t, x)\right) \quad+\sum_{s=1}^{l} \Delta^{s}\left(b_{s}(t, x) u^{m}(t, x)\right) \\
\quad \geq c(t, x) F(u(t, x)) \\
u^{m}(t, x)=\Delta\left(u^{m}(t, x)\right)=\cdots=\Delta^{l-1}\left(u^{m}(t, x)\right)=0 \\
x \in \partial \Omega \\
u \geq 0
\end{array}\right.
$$

where the boundary conditions is in the mean of trace.

Notice that this inequality is a special case of the above inequality. For the problem (1.2) we prove the nonexistence of nonnegative nontrivial global solutions for $F(u)=\left|\nabla u^{q}\right|^{p}$ when $p>2$ which is a better result with respect to the problem $(1.1)$.

Here, we describe the literature of the problems for the following problems:

$$
\begin{gathered}
\frac{\partial u}{\partial t}-\triangle u^{m}=\left|\nabla u^{q}\right|^{p}, u \geq 0, \\
\frac{\partial^{k} u}{\partial t^{k}}-\triangle u^{m}=|u|^{q}, u \geq 0, \\
\frac{\partial u}{\partial t}+\triangle^{2} u=\left|\nabla u^{q}\right|^{p}, u \geq 0, \\
\frac{\partial^{2} u}{\partial t^{2}}-\triangle u=|x|^{-\gamma}|u|^{q}, u \geq 0,
\end{gathered}
$$

These problems are special cases of (1.1) or (1.2).

For problem (1.3) Andreucci in [1] has proved the existence of local solution in $\mathbb{R}^{N}$ for $p q \geq m, m \geq 1,0<p<2$, and $N \geq 1$. In his work the initial data are measures.He proved the local solution for (1.4) in the supercritical case $q>m+2 / N$ and $k=1$. He discovered some useful inequalities for solutions in the paper.

Here we should mention that Souplet in [16] had proved existence of global solution for (1.4) when $k=m=1$ before Andreucci[1].

Problem (1.4) considered by Laptev in $[8,9]$. He has proved the nonexistence of nontrivial global solution for this problem whenever $\Omega$ is a cone or is a ball complement and nonnegative initial data.

Problem (1.5) with $q=1$ has considered by Bellout, Benachour and Titi in [2]. They used the Galerkin method to prove the short-time existence of weak and strong solutions for this initial boundary value problem.

Problem (1.6) has investigated by Pohozaev and Tesei in [14] when $\Omega$ is a smooth boundary domain such that $0 \in \Omega$. They show the instantaneous blow-up for this problem when the initial data has a critical point in 0 . They employ the Test Function method in their investigation.

For more information about the existence and nonexistence of solutions of nontrivial global solution for these problems the reader is refereed to $[3,4,5,6,7,10,11$, $12,13,16,17]$. 
2. General type inequalities. Consider $\Omega \subseteq \mathbb{R}^{N}$ is a domain with smooth boundary. Let $a_{i}(t, x), b_{\alpha}(t, x)$ be real measurable functions with upper bounds $A_{i}, B_{\alpha} \in \mathbb{R}^{+}$for all, $1 \leq i \leq k,|\alpha| \leq n$,respectively. As we mentioned before $a_{i}(t, x)$ is $i-1$ time differentiable at $t=0$, and let $c(t, x)$ be a real measurable function which has a lower bound $C>0$. Finally, let $\vec{A}=\left(A_{1}, \ldots, A_{k}\right)$ and $\mathbf{B}=\left\{B_{\alpha}\right\}_{|\alpha| \leq n}$.

Here we are interested to show that the following inequality has no nontrivial global solution on $(0, T), T \in(0,+\infty]$,for large values of initial data:

$$
\sum_{i=1}^{k} \frac{\partial^{i}}{\partial t^{i}}\left(a_{i}(t, x) u(t, x)\right)+\sum_{|\alpha| \leq n} D^{\alpha}\left(b_{\alpha}(t, x) u^{m_{\alpha}}(t, x)\right) \geq c(t, x) F(u(t, x)) .
$$

Here $F(u)=|u|^{q}$ or $|x|^{-\gamma}|u|^{q}$ or $\left|\nabla u^{q}\right|^{p}$ and $p, q>1$ are real numbers.For $F(u)=$ $|x|^{-\gamma}|u|^{q}$ we consider that $\Omega-\{0\}=\Omega$.

In the following we give the concept of solution.

Definition 2.1. Let $T \in(0,+\infty]$ and $\Omega \subseteq \mathbb{R}^{N}$ be an open set. By a global solution on $(0, T)$, we mean a function $u$ such that:

i) For $F(u)=|u|^{q}$ or $F(u)=|x|^{-\gamma}|u|^{q}$,

$$
u \in C\left([0, T) ; L_{\mathrm{loc}}^{q}(\Omega)\right) \bigcap\left(\bigcap_{|\alpha| \leq n} C\left([0, T) ; L_{\mathrm{loc}}^{m_{\alpha}}(\Omega)\right)\right) .
$$

ii) For $F(u)=\left|\nabla u^{q}\right|^{p}, u^{q}$ belongs to $C\left([0, T) ; H_{0}^{1}(\Omega)\right) \bigcap C\left([0, T) ; W_{0}^{1, p}(\Omega)\right)$ and $u \in \bigcap_{|\alpha| \leq n} C\left([0, T) ; L_{\mathrm{loc}}^{m_{\alpha}}(\Omega)\right)$.

Moreover,for every nonnegative function $\zeta(t, x) \in C_{t, x}^{\min \{n, k\}}([0, T] \times \Omega)$ and $\zeta(t,.) \in$ $C_{0}^{n}(\Omega)$ and $\zeta(., x) \in C^{k}[0, T]$ with $\zeta(T,)=.\cdots=\frac{\partial^{k-1}}{\partial t^{k-1}} \zeta(T,)=$.0 we have:

$$
\begin{aligned}
& \sum_{i=1}^{k} \sum_{j=1}^{i}(-1)^{j} \int_{\Omega} \frac{\partial^{i-j}\left(a_{i}(0, x) u(0, x)\right)}{\partial t^{i-j}} \frac{\partial^{j-1} \zeta(0, x)}{\partial t^{j-1}} d x \\
& +\sum_{j=1}^{k}(-1)^{j} \int_{0}^{T} \int_{\Omega} a_{j}(t, x) u(t, x) \frac{\partial^{j} \zeta(t, x)}{\partial t^{j}} d x d t \\
& +(-1)^{|\alpha|} \sum_{|\alpha| \leq n} \int_{0}^{T} \int_{\Omega} b_{\alpha}(t, x) u^{m_{\alpha}}(t, x) D_{x}^{\alpha}(\zeta(t, x)) d x d t \\
& \geq \int_{0}^{T} \int_{\Omega} c(t, x) F(u(t, x)) \zeta(t, x) d x d t,
\end{aligned}
$$

where all the integrals are exist. Let $\phi$ be the first eigenvector of $-\Delta$ in $H_{0}^{1}(\Omega)$ such that $\phi>0$, and $\int_{\Omega} \phi=1$ and:

$$
\psi(x):=\phi^{\sigma}(x),
$$

where $\sigma>n$ is a real number. 
On the other hand let $\beta=k q^{\prime}+1$ and define $c_{\beta, l}$ as follow:

$$
\begin{gathered}
c_{\beta .0}=1, \\
c_{\beta, l}=\beta(\beta-1) \ldots(\beta-l+1),
\end{gathered}
$$

for $l \leq \beta$.

We have the following theorem related to inequality (2.1).

TheOREm 2.2. Let $\Omega$ be a bounded smooth domain in $\mathbb{R}^{N}$ and $q>m_{\alpha}$ for all $|\alpha| \leq n$, and $\tau>0$.

a) Let $F(u)=|u|^{q}$ and

$$
\sigma=\left[\max _{|\alpha| \leq n}\left\{\frac{q}{q-m_{\alpha}}|\alpha|-1, n\right\}\right]+1
$$

Then, there exists a positive number $M_{1}=M_{1}(\Omega, \tau, \vec{A}, \mathbf{B}, C, k, n, N)$ such that $(2.2)$ has no nontrivial global solution on $(0, \tau)$ when:

$$
\sum_{i=1}^{k} \sum_{j=1}^{i} c_{\beta, j-1} \tau^{\beta-j+1} \int_{\Omega} \frac{\partial^{i-j}\left(a_{i}(0, x) u(0, x)\right)}{\partial^{i-j} t} \psi(x) d x>M_{1}
$$

b) Let $F(u)=\left|\nabla u^{q}\right|^{p}, p-1>n+1$ and $p-1>\sigma>n+1$ a real fixed number. Then, there exists a positive number $M_{2}=M_{2}(\Omega, \tau, \vec{A}, \mathbf{B}, C, k, n, N)$ such that (2.2) has no nonnegative nontrivial global solution on $(0, \tau)$ when:

$$
\sum_{i=1}^{k} \sum_{j=1}^{i} c_{\beta, j-1} \tau^{\beta-j+1} \int_{\Omega} \frac{\partial^{i-j}\left(a_{i}(0, x) u(0, x)\right)}{\partial^{i-j} t} \psi(x) d x>M_{2}
$$

In order to prove this theorem, we need the following lemma from[15].

Lemma 2.3. Suppose that $\Omega \subseteq \mathbb{R}^{N}$ is a bounded and $C^{2}$ domain and $\phi$ is the first eigenvector of the operator $-\Delta$ in $H_{0}^{1}(\Omega)$. Then

$$
C(\phi, \Omega):=\int_{\Omega} \frac{1}{\phi^{\alpha}}<\infty, \quad \forall \alpha \in(-\infty, 1) .
$$

Proof of Theorem 2.2. For $0<\tau<T$ set

$$
\hat{\phi}(t):= \begin{cases}(\tau-t)^{\beta} & 0 \leq t \leq \tau \\ 0 & \tau \leq t \leq T\end{cases}
$$

from the definition of $\beta$ we know that:

$\left.i^{\prime}\right) \beta-i q^{\prime}+1>1$ for all $1 \leq i \leq k$,

$\left.i i^{\prime}\right) \beta \geq k+1$,

where $q^{\prime}$ is the conjugate of $q$. Let

$$
\zeta(t, x):=\hat{\phi}(t) \psi(x) .
$$


By using this $\zeta(t, x)$ in Definition 2.1, and by considering definition of $\sigma$ and $\beta$ we get:

$$
\begin{aligned}
& -\sum_{i=1}^{k} \sum_{j=1}^{i} \tau^{\beta-j+1} c_{\beta, j-1} \int_{\Omega} \frac{\partial^{i-j}\left(a_{i}(0, x) u(0, x)\right)}{\partial^{i-j} t} \psi(x) d x \\
& +\sum_{i=1}^{k} \int_{0}^{\tau} \int_{\Omega} c_{\beta, i}(\tau-t)^{\beta-i} a_{i}(t, x) u(t, x) \psi(x) d x d t \\
& +\sum_{|\alpha| \leq n}(-1)^{|\alpha|} \int_{0}^{\tau} \int_{\Omega}(\tau-t)^{\beta} b_{\alpha}(t, x) u^{m_{\alpha}}(t, x) D^{\alpha} \psi(x) d x d t \\
& \geq \int_{0}^{\tau} \int_{\Omega} c(t, x)(\tau-t)^{\beta} F(u(t, x)) \psi(x) d x d t .
\end{aligned}
$$

Proof of part (a). Consider $u(t, x)$ as a solution.Let $K>0$ be a positive real number. By Holder's and Young's inequalities on $(0, \tau) \times \Omega$ from $\left|a_{i}(t, x)\right| \leq A_{i}, 1 \leq$ $i \leq k$ we get:

$$
\begin{aligned}
& \int_{0}^{\tau} \int_{\Omega} c_{\beta, i}(\tau-t)^{\beta-i} a_{i}(t, x) u(t, x) \psi(x) d x d t \\
& \leq \int_{0}^{\tau} \int_{\Omega} K(\tau-t)^{\frac{\beta}{q}} \psi^{\frac{1}{q}}(x)|u(t, x)| K^{-1} A_{i} c_{\beta, i}(\tau-t)^{\frac{\beta}{q^{\prime}}-i} \psi^{\frac{1}{q^{\prime}}}(x) d x d t \\
& \leq \frac{K^{q}}{q} \int_{0}^{\tau} \int_{\Omega}(\tau-t)^{\beta}|u(t, x)|^{q} \psi(x) d x d t \\
& +\frac{c_{\beta, i}^{q^{\prime}} A_{i}^{q^{\prime}}}{q^{\prime} K^{q^{\prime}}} C(\psi) \frac{\tau^{\beta-i q^{\prime}+1}}{\beta-i q^{\prime}+1},
\end{aligned}
$$

where $C(\psi):=\int_{\Omega} \psi(x) d x$.

On the other hand, let $K_{\alpha}$ be a positive number and $r_{\alpha}=\frac{q}{m_{\alpha}}$. By applying Holder's and Young's inequalities on $\Omega$ from $\left|b_{\alpha}(t, x)\right| \leq B_{\alpha}$ we get:

$$
\begin{aligned}
& \int_{0}^{\tau} \int_{\Omega}(\tau-t)^{\beta} b_{\alpha}(t, x) u^{m_{\alpha}}(t, x) D^{\alpha} \psi(x) d x \\
& \leq \int_{0}^{\tau} \int_{\Omega}(\tau-t)^{\beta} K_{\alpha}|u(t, x)|^{m_{\alpha}} \psi^{\frac{1}{r_{\alpha}}}(x) K_{\alpha}^{-1} B_{\alpha} \psi^{-\frac{1}{r_{\alpha}}}(x) D^{\alpha} \psi(x) d x \\
& \leq \frac{K_{\alpha}^{r_{\alpha}}}{r_{\alpha}} \int_{0}^{\tau} \int_{\Omega}(\tau-t)^{\beta}|u(t, x)|^{q} \psi(x)+\frac{B_{\alpha}^{r_{\alpha}^{\prime}} \tau^{\beta+1}}{r_{\alpha}^{\prime} K_{\alpha}^{r_{\alpha}^{\prime}}(\beta+1)} \int_{\Omega} \frac{\left|D^{\alpha} \psi(x)\right|^{r_{\alpha}^{\prime}}}{\psi^{\frac{r_{\alpha}^{\alpha}}{r_{\alpha}}}} d x .
\end{aligned}
$$

Now we will show that the last integral exists. We know:

$$
\sigma=\left[\max _{|\alpha| \leq n}\left\{\frac{q}{q-m_{\alpha}}|\alpha|-1, n\right\}\right]+1 .
$$

Note that $\left|D^{\alpha} \psi(x)\right|^{r_{\alpha}^{\prime}}=|P(x)|^{r_{\alpha}^{\prime}} \phi^{(\sigma-|\alpha|) r_{\alpha}^{\prime}}(x)$ when $P$ is a polynomial of $\phi$ and its partial derivations. A simple calculation shows that:

$$
\sigma \frac{r_{\alpha}^{\prime}}{r_{\alpha}}-(\sigma-|\alpha|) r_{\alpha}^{\prime}<1
$$


Therefore, $\frac{\left|D^{\alpha} \psi(x)\right|^{\prime}}{\psi^{\frac{r_{\alpha}^{\prime}}{r_{\alpha}}}}=\frac{|P(x)|_{\alpha}^{r_{\alpha}^{\prime}}}{\phi^{\frac{r_{\alpha}^{\prime}}{r_{\alpha}} \sigma-(\sigma-|\alpha|) r_{\alpha}^{\prime}}}$ is integrable, by Lemma 2.3; indeed:

$$
C_{\alpha}(\psi)=\int_{\Omega} \frac{\left|D^{\alpha} \psi(x)\right|^{r_{\alpha}^{\prime}}}{\psi^{\frac{r_{\alpha}^{\prime}}{r_{\alpha}}}}<\infty .
$$

It is notable that by $\phi=-\lambda \triangle \phi$ and Relich's Theorem $\phi$ and its derivations are bounded on $\Omega$. ( $1 / \lambda$ is the eigenvalue due to $-\Delta)$

By using (2.6) and (2.7) in (2.5) for $F(u)=|u|^{q}$ from the assumption $c(t, x) \geq C$, we get:

$$
\begin{aligned}
& -\sum_{i=1}^{k} \sum_{j=1}^{i} c_{\beta, j-1} \tau^{\beta-j+1} \int_{\Omega} \frac{\partial^{i-j}\left(a_{i}(0, x) u(0, x)\right)}{\partial^{i-j} t} \psi(x) d x \\
& +\sum_{i=1}^{k} \frac{c_{\beta, i}^{q^{\prime}} A_{i}^{q^{\prime}}}{q^{\prime} K^{q^{\prime}}} C(\psi) \frac{\tau^{\beta-i q^{\prime}+1}}{\beta-i q^{\prime}+1}+\sum_{|\alpha| \leq n} \frac{B_{\alpha}^{r_{\alpha}^{\prime}}}{r_{\alpha}^{\prime} K_{\alpha}^{r_{\alpha}^{\prime}}} C_{\alpha}(\psi) \frac{\tau^{\beta+1}}{\beta+1} \\
& \geq\left(C-\frac{k K^{q}}{q}-\sum_{|\alpha| \leq n} \frac{K_{\alpha}^{r_{\alpha}}}{r_{\alpha}}\right) \int_{0}^{\tau} \int_{\Omega}|u(t, x)|^{q} \psi(x)(\tau-t)^{\beta} d x d t .
\end{aligned}
$$

Now $K>0$ and $K_{\alpha}>0,|\alpha| \leq n$, can be chosen so that $C-\frac{k K^{q}}{q}-\sum_{|\alpha| \leq n} \frac{K_{\alpha}^{r_{\alpha}}}{r_{\alpha}} \geq 0$. Let:

$$
M_{1}=\sum_{i=1}^{k} \frac{c_{\beta, i}^{q^{\prime}} A_{i}^{q^{\prime}}}{q^{\prime} K^{q^{\prime}}} C(\psi) \frac{\tau^{\beta-i q^{\prime}+1}}{\beta-i q^{\prime}+1}+\sum_{|\alpha| \leq n} \frac{B_{\alpha}^{r_{\alpha}^{\prime}}}{r_{\alpha}^{\prime} K_{\alpha}^{r_{\alpha}^{\prime}}} C_{\alpha}(\psi) \frac{\tau^{\beta+1}}{\beta+1} .
$$

If $\sum_{i=1}^{k} \sum_{j=1}^{i} c_{\beta, j-1} \tau^{\beta-j+1} \int_{\Omega} \frac{\partial^{i-j}\left(a_{i}(0, x) u(0, x)\right)}{\partial^{i-j} t} \psi(x) d x>M_{1}$ then the left hand side of (2.8) will be negative which is contradiction. This complete the proof of part (a). प

Proof of part (b). The proof of this part is similar. Since the estimations in the proof are crucial we give the details of the proof. Let $u(t, x)$ be a nonnegative solution. For every $K>0$, by Holder's and Young's inequalities on $(0, \tau) \times \Omega$ and $\left|a_{i}(t, x)\right| \leq A_{i}, 1 \leq i \leq k$ we get:

$$
\begin{aligned}
& \int_{0}^{\tau} \int_{\Omega} c_{\beta, i}(\tau-t)^{\beta-i} a_{i}(t, x) u(t, x) \psi(x) d x d t \\
& \leq \int_{0}^{\tau} \int_{\Omega} K(\tau-t)^{\frac{\beta}{q}} \phi^{\frac{1}{q}}(x) u(t, x) K^{-1} c_{\beta, i} A_{i} \phi^{-\frac{1}{q}}(x)(\tau-t)^{\frac{\beta}{q^{\prime}}-i} \psi(x) d x d t \\
& \leq \frac{K^{q}}{q} \int_{0}^{\tau} \int_{\Omega}(\tau-t)^{\beta} u^{q}(t, x) \phi(x)+\frac{c_{\beta, i}^{q^{\prime}} A_{i}^{q^{\prime}}}{q^{\prime} K^{q^{\prime}}} \frac{\tau^{\beta-i q^{\prime}+1}}{\beta-i q^{\prime}+1} \int_{\Omega} \frac{\psi^{q^{\prime}}(x)}{\phi^{\frac{q^{\prime}}{q}}(x)} d x .
\end{aligned}
$$

Note that by $\frac{q^{\prime}}{q}-\sigma q^{\prime}<1$, for all $\sigma \geq 1$, and Lemma (2.3) we have

$$
D_{1}(\phi)=\int_{\Omega} \frac{\psi^{q^{\prime}}(x)}{\phi^{\frac{q^{\prime}}{q}}(x)}<\infty
$$


Now assume that $\lambda>0$ is a positive number such that $1 / \lambda$ is the eigenvalue corresponding to $\phi$. Then by condition $\left.u(t, x)\right|_{\partial \Omega} \equiv 0$, Holder's and Young's inequality on $\Omega$ we get:

$$
\begin{aligned}
& \int_{\Omega} u^{q}(t, x) \phi(x)=\lambda \int_{\Omega} \nabla u^{q}(t, x) \cdot \nabla \phi(x) d x \\
& \leq \lambda \int_{\Omega}\left|\nabla u^{q}(t, x)\right| \psi^{\frac{1}{p}}(x) \psi^{-\frac{1}{p}}(x)|\nabla \phi(x)| d x \\
& \leq \frac{\lambda}{p} \int_{\Omega}\left|\nabla u^{q}(t, x)\right|^{p} \psi(x) d x+\frac{\lambda}{p^{\prime}} \int_{\Omega} \frac{|\nabla \phi(x)|^{p^{\prime}}}{\psi^{\frac{p^{\prime}}{p}}(x)} d x .
\end{aligned}
$$

Note that by conditions of part (b) of the theorem we have $p-1>n+1$. We know $\sigma \geq 0$ is such that $n+1 \leq \sigma<p-1$. Therfore, by Lemma 2.3 we obtain:

$$
D_{2}(\phi):=\int_{\Omega} \frac{|\nabla \phi(x)|^{p^{\prime}}}{\psi^{\frac{p^{\prime}}{p}}(x)} d x<\infty .
$$

Now (2.10) and (2.9) imply:

$$
\begin{aligned}
& \int_{0}^{\tau} \int_{\Omega} c_{\beta, i}(\tau-t)^{\beta-i} a_{i}(t, x) u(t, x) \psi(x) d x d t \\
& \leq \frac{K^{q} \lambda}{q p} \int_{0}^{\tau} \int_{\Omega}(\tau-t)^{\beta}\left|\nabla u^{q}(t, x)\right|^{p} \psi(x) d x+\frac{K^{q} \lambda \tau^{\beta+1}}{q p^{\prime}(\beta+1)} D_{2}(\phi) \\
& +\frac{c_{\beta, i}^{q^{\prime}} A_{i}^{q^{\prime}}}{q^{\prime} K^{q^{\prime}}} \frac{\tau^{\beta-i q^{\prime}+1}}{\beta-i q^{\prime}+1} D_{1}(\phi) .
\end{aligned}
$$

On the other hand, for any positive number $K_{\alpha}$ and $r_{\alpha}=\frac{q}{m_{\alpha}}$, by Holder's and Young's inequality on $\Omega$ we get:

$$
\begin{aligned}
& \int_{0}^{\tau} \int_{\Omega}(\tau-t)^{\beta} b_{\alpha}(t, x) u^{m_{\alpha}}(t, x) D^{\alpha} \psi(x) d x \\
& \leq \int_{0}^{\tau} \int_{\Omega}(\tau-t)^{\beta} K_{\alpha} u^{m_{\alpha}}(t, x) \phi^{\frac{1}{r_{\alpha}}}(x) \phi^{-\frac{1}{r_{\alpha}}}(x) K_{\alpha}^{-1} B_{\alpha} D^{\alpha} \psi(x) d x d t \\
& \leq \frac{K_{\alpha}^{r_{\alpha}}}{r_{\alpha}} \int_{0}^{\tau} \int_{\Omega}(\tau-t)^{\beta} u^{q}(t, x) \phi(x) d x d t+\frac{B_{\alpha}^{r_{\alpha}^{\prime}} \tau^{\beta+1}}{r_{\alpha}^{\prime} K_{\alpha}^{r_{\alpha}^{\prime}}(\beta+1)} \int_{\Omega} \frac{\left|D^{\alpha} \psi(x)\right|^{r_{\alpha}^{\prime}}}{\phi(x)^{\frac{r_{\alpha}^{\prime}}{r_{\alpha}}}} .
\end{aligned}
$$

Note that from $\sigma \geq n+1$ we have $\sigma \geq|\alpha|+1$ for all $|\alpha| \leq n$. Morover, from $q>m_{\alpha}$ we get $\sigma>\frac{2 m_{\alpha}}{q}+|\alpha|-1$. This implies the inequality $\frac{r_{\alpha}^{\prime}}{r_{\alpha}}-(\sigma-|\alpha|) r_{\alpha}^{\prime}<1$. So we have:

$$
D_{3}(\phi, \alpha):=\int_{\Omega} \frac{\left|D^{\alpha} \psi(x)\right|^{r_{\alpha}^{\prime}}}{\phi(x)^{\frac{r_{\alpha}^{\prime}}{r_{\alpha}}}} d x<\infty .
$$


Again from condition $\left.u(t, x)\right|_{\partial \Omega} \equiv 0$, and by Holder's and Young's inequalities on $\Omega$ and the definition of $\phi$ we get:

$$
\begin{aligned}
& \int_{0}^{\tau} \int_{\Omega}(\tau-t)^{\beta} u^{q}(t, x) \phi(x)=\lambda \int_{0}^{\tau} \int_{\Omega}(\tau-t)^{\beta}\left(\nabla u^{q}(t, x)\right) \cdot(\nabla \phi(x)) d x d t \\
& \leq \lambda \int_{0}^{\tau} \int_{\Omega}(\tau-t)^{\beta}\left|\nabla u^{q}(t, x)\right| \psi(x)^{\frac{1}{p}} \psi(x)^{-\frac{1}{p}}|\nabla \phi(x)| d x d t \\
& \leq \frac{\lambda}{p} \int_{0}^{\tau} \int_{\Omega}(\tau-t)^{\beta}\left|\nabla u^{q}(t, x)\right|^{p} \psi(x) d x d t+\frac{\lambda}{p^{\prime}} \frac{\tau^{\beta+1}}{\beta+1} \int_{\Omega} \frac{|\nabla \phi(x)|^{p^{\prime}}}{\psi^{\frac{p^{\prime}}{p}}(x)} .
\end{aligned}
$$

Note that we have chosen $\sigma$ so that $\sigma<p-1$ or $\frac{\sigma p^{\prime}}{p}<1$, we must have:

$$
D_{2}(\phi)=\int_{\Omega} \frac{|\nabla \phi(x)|^{p^{\prime}}}{\psi(x)^{\frac{p^{\prime}}{p}}} d x<\infty
$$

Substitute (2.13) into (2.12) to get:

$$
\begin{aligned}
& \int_{0}^{\tau} \int_{\Omega}(\tau-t)^{\beta} b_{\alpha}(t, x) u^{m_{\alpha}}(t, x) D^{\alpha} \psi(x) d x \\
& \leq \frac{K_{\alpha}^{r_{\alpha}} \lambda}{r_{\alpha} p} \int_{0}^{\tau} \int_{\Omega}(\tau-t)^{\beta}\left|\nabla u^{q}(t, x)\right|^{p} \psi(x) d x+\frac{K_{\alpha}^{r_{\alpha}} \lambda \tau^{\beta+1}}{r_{\alpha} p^{\prime}(\beta+1)} D_{2}(\phi) \\
& +\frac{B_{\alpha}^{r_{\alpha}^{\prime}} \tau^{\beta+1}}{r_{\alpha}^{\prime} K_{\alpha}^{r_{\alpha}^{\prime}}(\beta+1)} D_{3}(\phi, \alpha) .
\end{aligned}
$$

By using (2.11) in (2.5) for $F(u)=\left|\nabla u^{q}\right|^{p}$ we get:

$$
\begin{aligned}
& -\sum_{i=1}^{k} \sum_{j=i}^{i} \tau^{\beta-j+1} c_{\beta, j-1} \int_{\Omega} \frac{\partial^{i-j}\left(a_{i}(0, x) u(0, x)\right)}{\partial t^{i-j}} \psi(x) d x \\
& +\sum_{i=1}^{k} \frac{c_{\beta, i}^{q^{\prime}} A_{i}^{q^{\prime}}}{q^{\prime} K^{q^{\prime}}} \frac{\tau^{\beta-i q^{\prime}+1}}{\beta-i q^{\prime}+1} D_{1}(\phi)+\frac{k K^{q} \lambda \tau^{\beta+1}}{q p^{\prime}(\beta+1)} D_{2}(\phi) \\
& +\sum_{|\alpha| \leq n}\left(\frac{K_{\alpha}^{r_{\alpha}} \lambda}{r_{\alpha} p^{\prime}} D_{2}(\phi)+\frac{B_{\alpha}^{r_{\alpha}^{\prime}}}{r_{\alpha}^{\prime} K_{\alpha}^{r_{\alpha}^{\prime}}} D_{3}(\phi, \alpha)\right) \frac{\tau^{\beta+1}}{\beta+1} \\
& \geq\left(C-\frac{k K^{q} \lambda}{q p}-\sum_{|\alpha| \leq n} \frac{K_{\alpha}^{r_{\alpha}} \lambda}{r_{\alpha} p}\right) \int_{0}^{\tau} \int_{\Omega}\left|\nabla u^{q}(t, x)\right|^{p} \psi(x) d x d t .
\end{aligned}
$$

Notice that by choosing $K$ and $K_{\alpha}(|\alpha| \leq n)$, very small positive number we have:

$$
C-\frac{k K^{q} \lambda}{q p}-\sum_{|\alpha| \leq n} \frac{K_{\alpha}^{r_{\alpha}} \lambda}{r_{\alpha} p} \geq 0
$$


Now let:

$$
\begin{aligned}
M_{2}= & \sum_{i=1}^{k} \frac{c_{\beta, i}^{q^{\prime}} A_{i}^{q^{\prime}}}{q^{\prime} K^{q^{\prime}}} \frac{\tau^{\beta-i q^{\prime}+1}}{\beta-i q^{\prime}+1} D_{1}(\phi)+\frac{k K^{q} \lambda \tau^{\beta+1}}{q p^{\prime}(\beta+1)} D_{2}(\phi) \\
& +\sum_{|\alpha| \leq n}\left(\frac{K_{\alpha}^{r_{\alpha}} \lambda}{r_{\alpha} p^{\prime}} D_{2}(\phi)+\frac{B_{\alpha}^{r_{\alpha}^{\prime}}}{r_{\alpha}^{\prime} K_{\alpha}^{r_{\alpha}^{\prime}}} D_{3}(\phi, \alpha)\right) \frac{\tau^{\beta+1}}{\beta+1}
\end{aligned}
$$

Therefore, if $\sum_{i=1}^{k} \sum_{j=i}^{i} \tau^{\beta-j+1} c_{\beta, j-1} \int_{\Omega} \frac{\partial^{i-j}\left(a_{i}(0, x) u(0, x)\right)}{\partial t^{i-j}} \psi(x) d x>M_{2}$; then, the left hand side of (2.15) will be negative. This is a contradiction.

We now want to discuss about solution in $\mathbb{R}^{N}$. Let $\Omega_{d}:=B(0, d)$. Now we apply the argument of part $(a)$ for $\Omega=\Omega_{d}$. Similar to(2.8), we have:

$$
\begin{aligned}
& -\sum_{i=1}^{k} \sum_{j=1}^{i} c_{\beta, j-1} \tau^{\beta-j+1} \int_{\Omega_{d}} \frac{\partial^{i-j}\left(a_{i}(0, x) u(0, x)\right)}{\partial^{i-j} t} \psi_{d}(x) d x \\
& +\sum_{i=1}^{k} \frac{c_{\beta, i}^{q^{\prime}} A_{i}^{q^{\prime}}}{q^{\prime} K^{q^{\prime}}} C\left(\psi_{d}\right) \frac{\tau^{\beta-i q^{\prime}+1}}{\beta-i q^{\prime}+1}+\sum_{|\alpha| \leq n} \frac{B_{\alpha}^{r_{\alpha}^{\prime}}}{r_{\alpha}^{\prime} K_{\alpha}^{r_{\alpha}^{\prime}}} C_{\alpha}\left(\psi_{d}\right) \frac{\tau^{\beta+1}}{\beta+1} \\
& \geq\left(C-\frac{k K^{q}}{q}-\sum_{|\alpha| \leq n} \frac{K_{\alpha}^{r_{\alpha}}}{r_{\alpha}}\right) \int_{0}^{\tau} \int_{\Omega_{d}}|u(t, x)|^{q} \psi_{d}(x)(\tau-t)^{\beta} d x d t,
\end{aligned}
$$

where $\phi_{d}(x):=\phi(x / d)$ and $\psi_{d}(x):=\psi(x / d), x \in \Omega_{d}$ and $\phi>0$ is the first eigenvector of $-\triangle$ in $H_{0}^{1}\left(B_{1}(\Omega)\right)$. We can easily see that

$$
\begin{aligned}
C_{\alpha}\left(\psi_{d}\right)=\int_{\Omega_{d}} \frac{\left|D^{\alpha} \psi_{d}(x)\right|_{\alpha}^{r_{\alpha}^{\prime}}}{\psi_{d}(x)^{\frac{r_{\alpha}^{\prime}}{r_{\alpha}}}} d x & =d^{-|\alpha| r_{\alpha}^{\prime}+N} \int_{\Omega_{d}} \frac{\left|D^{\alpha} \psi(x / d)\right|^{r_{\alpha}^{\prime}}}{\psi^{\frac{r_{\alpha}^{\prime}}{r_{\alpha}}}(x / d)} d(x / d), \\
& =d^{-|\alpha| r_{\alpha}^{\prime}+N} L(\Omega)
\end{aligned}
$$

where $L(\Omega, \alpha)=\int_{\Omega_{1}} \frac{\left|D^{\alpha} \psi\right|^{r_{\alpha}^{\prime}}}{\psi_{\alpha}^{r_{\alpha}^{\prime}}(x)} d x$.

On the other hand, we have:

$$
C\left(\psi_{d}\right)=\int_{\Omega_{d}} \psi_{d}(x) d x=d^{N} L(\Omega)
$$

where

$$
L(\Omega)=\int_{\Omega_{1}} \psi(x) d x
$$

and

$$
\int_{\Omega_{d}} \frac{\partial^{i-j}\left(a_{i}(0, x) u(0, x)\right)}{\partial^{i-j} t} \psi_{d}(x) d x=d^{N} \int_{\Omega_{1}} \frac{\partial^{i-j}\left(a_{i}(0, d x) u(0, d x)\right)}{\partial^{i-j} t} \psi(x) d x .
$$


Now we deduce from (2.16), (2.17), (2.18) and (2.19)

$$
\begin{aligned}
& -\sum_{i=1}^{k} \sum_{j=1}^{i} c_{\beta, j-1} \tau^{\beta-j+1} d^{N} \int_{\Omega_{1}} \frac{\partial^{i-j}\left(a_{i}(0, d x) u(0, d x)\right)}{\partial^{i-j} t} \psi(x) d x \\
& +\sum_{i=1}^{k} \frac{c_{\beta, i}^{q^{\prime}} A_{i}^{q^{\prime}} d^{N}}{q^{\prime} c^{q^{\prime}} K^{q^{\prime}}} L(\Omega) \frac{\tau^{\beta-i q^{\prime}+1}}{\beta-i q^{\prime}+1}+\sum_{|\alpha| \leq n} d^{-|\alpha| r_{\alpha}^{\prime}+N} \frac{B_{\alpha}^{r_{\alpha}^{\prime}}}{r_{\alpha}^{\prime} K_{\alpha}^{r_{\alpha}^{\prime}}} L(\alpha, \Omega) \frac{\tau^{\beta+1}}{\beta+1} \\
& \geq 0 .
\end{aligned}
$$

Consider that $j_{1}:=\min \left\{j \leq k ; \exists i \geq j\right.$ s.t. $\lim _{\sup } \operatorname{su}_{d \rightarrow \infty} \int_{\Omega_{1}} \frac{\partial^{i-j_{1}}\left(a_{i}(0, d x) u(0, d x)\right)}{\partial^{i-j_{1} t}} \psi(x) d x$ $>0\}$. Divide (2.20) by $d^{N}$ and then let $d \rightarrow \infty$ so we get

$$
\begin{aligned}
& -c_{\beta, j_{1}-1} \tau^{\beta-j_{1}+1} \limsup _{d \rightarrow \infty} \int_{\Omega_{1}} \frac{\partial^{i-j_{1}}\left(a_{i}(0, d x) u(0, d x)\right)}{\partial^{i-j_{1}} t} \psi(x) d x \\
& +\sum_{i=1}^{k} \frac{c_{\beta, i}^{q^{\prime}} A_{i}^{q^{\prime}}}{q^{\prime} K^{q^{\prime}}} L(\Omega) \frac{\tau^{\beta-i q^{\prime}+1}}{\beta-i q^{\prime}+1} \geq 0 .
\end{aligned}
$$

Now if $q^{\prime}>j_{1}$, by letting $\tau \rightarrow \infty$, the left hand side of (2.21) will be negative which is a contradiction.

On the other hand, let $i_{1}=\min \left\{i ; a_{i}(t, x) \neq 0\right\}$ and $e=\min \left\{|\alpha| r_{\alpha}^{\prime} ; b_{\alpha}(t, x) \neq 0\right\}$. Divide (2.16) with $\tau^{\beta}$; then, by (2.17) and (2.18) we obtain:

$$
\begin{aligned}
& -\sum_{i=1}^{k} \sum_{j=1}^{i} c_{\beta, j-1} \tau^{-j+1} \int_{\Omega_{d}} \frac{\partial^{i-j}\left(a_{i}(0, x) u(0, x)\right)}{\partial^{i-j} t} \psi(x) d x \\
& +\sum_{i=1}^{k} \frac{c_{\beta, i}^{q^{\prime}} A_{i}^{q^{\prime}}}{q^{\prime} c^{\frac{q^{\prime}}{q}} K^{\frac{q^{\prime}}{q}}} d^{N} L(\Omega) \frac{\tau^{-i q^{\prime}+1}}{-i q^{\prime}+1}+\sum_{|\alpha| \leq n} d^{-|\alpha| r_{\alpha}^{\prime}+N} \frac{B_{\alpha}^{r_{\alpha}^{\prime}}}{r_{\alpha}^{\prime} K_{\alpha}^{r_{\alpha}^{\prime}}} L(\alpha, \Omega) \frac{\tau}{\beta+1} \\
& \geq \psi_{1 / 2}(1 / 2)^{\beta}\left(C-\frac{k K^{q}}{q}-\sum_{|\alpha| \leq n} \frac{K_{\alpha}^{r_{\alpha}}}{r_{\alpha}}\right) \int_{0}^{\tau / 2} \int_{\Omega_{d / 2}}|u(t, x)|^{q} d x d t .
\end{aligned}
$$

where $\psi_{1 / 2}=\psi(1 / 2,0, \ldots, 0)$. Now suppose that $e>N$ and $\frac{1}{e-N}<\frac{i_{1} q^{\prime}-1}{N}$ and initial data are nonnegative. Assume $A>0$ is a number such that $\frac{1}{e-N}<A<\frac{i_{1} q^{\prime}-1}{N}$. Let $d=\tau^{A}$. Since $-i_{1} q^{\prime}+1+N A<0$ and $(-e+N) A+1<0$ by letting $\tau \rightarrow \infty$ in $(2.22)$ we get $\int_{0}^{\infty} \int_{\mathbb{R}^{N}}|u|^{q} \leq 0$. Then $u \equiv 0$.

We have the following theorem:

TheOrem 2.4. Let $\Omega=\mathbb{R}^{N}, i_{1}$, and $j_{1}$ be as above. Suppose that the initial data are positive. Then if $q^{\prime}>j_{1}$ or $e>N$ and $\frac{1}{e-N}<\frac{i_{1} q^{\prime}-1}{N}$; then, we have no global solution on $[0,+\infty)$. 
Now consider the following problem.

$$
\begin{cases}\sum_{i=1}^{k} \frac{\partial^{i}}{\partial t^{i}}\left(a_{i}(t, x) u(t, x)\right) & +\sum_{|\alpha| \leq n} D^{\alpha}\left(b_{\alpha}(t, x) u^{m_{\alpha}}(t, x)\right), \\ & \geq|x|^{-\gamma}|u|^{q} \\ x \in \Omega \backslash\{0\} & \end{cases}
$$

where $\gamma>0$ is a real number and other things as above. In this case some of the initial data have critical in zero.

Let $x_{\epsilon}=(0, \ldots, 0,2 \epsilon)$ and $\Omega_{\epsilon}=B_{\epsilon}\left(x_{\epsilon}\right)$ and consider for $\epsilon<\epsilon_{0}, B_{\epsilon}\left(x_{\epsilon}\right) \subseteq \Omega$.

Let $u$ be a solution for (2.23) then, it is easy to see that $u$ is a solution of

$$
\left\{\begin{aligned}
\sum_{i=1}^{k} \frac{\partial^{i}}{\partial t^{i}}\left(a_{i}(t, x) u(t, x)\right) & +\sum_{|\alpha| \leq n} D^{\alpha}\left(b_{\alpha}(t, x) u^{m_{\alpha}}(t, x)\right), \\
& \geq \epsilon^{-\gamma}|u|^{q} \\
x \in \Omega_{\epsilon} . &
\end{aligned}\right.
$$

By the same argument of above we have:

$$
\begin{aligned}
& -\sum_{i=1}^{k} \sum_{j=1}^{i} c_{\beta, j-1} \tau^{\beta-j+1} \int_{\Omega_{\epsilon}} \frac{\partial^{i-j}\left(a_{i}(0, x) u(0, x)\right)}{\partial^{i-j} t} \psi_{\epsilon}(x) d x \\
& +\sum_{i=1}^{k} \frac{c_{\beta, i}^{q^{\prime}} A_{i}^{q^{\prime}}}{q^{\prime} K^{\frac{q^{\prime}}{q}}} C\left(\psi_{\epsilon}\right) \frac{\tau^{\beta-i q^{\prime}+1}}{\beta-i q^{\prime}+1}+\sum_{|\alpha| \leq n} \frac{B_{\alpha}^{r_{\alpha}^{\prime}}}{r_{\alpha}^{\prime} K_{\alpha}^{r_{\alpha}^{\prime}}} C_{\alpha}\left(\psi_{\epsilon}\right) \frac{\tau^{\beta+1}}{\beta+1} \\
& \geq\left(\epsilon^{-\gamma}-\frac{k K^{q}}{q}-\sum_{|\alpha| \leq n} \frac{K_{\alpha}^{r_{\alpha}}}{r_{\alpha}}\right) \int_{0}^{\tau} \int_{\Omega_{\epsilon}}|u(t, x)|^{q} \psi_{\epsilon}(x)(\tau-t)^{\beta} d x d t .
\end{aligned}
$$

where $\phi$ is the first eigenvector of $-\Delta$ in $H_{0}^{1}\left(B_{1}(0)\right)$ and $\phi_{\epsilon}(x)=\phi\left((1 / \epsilon)\left(x-x_{\epsilon}\right)\right)$. Now we let $K^{q}=K^{r_{\alpha}}=d \epsilon^{-\gamma}$ where $d$ is so small that such that:

$$
1-d \frac{k}{q}+d \sum_{|\alpha| \leq n} \frac{1}{r_{\alpha}}>0
$$

We have:

$$
C\left(\psi_{\epsilon}\right)=\epsilon^{N} L(\Omega)
$$

and

$$
C_{\alpha}\left(\psi_{\epsilon}\right)=\epsilon^{N-|\alpha| r_{\alpha}^{\prime}} L(\alpha, \Omega)
$$

and

$$
\int_{\Omega_{\epsilon}} \frac{\partial^{i-j}\left(a_{i}(0, x) u(0, x)\right)}{\partial^{i-j} t} \psi_{\epsilon}(x) d x=\epsilon^{N} \int_{\Omega} \frac{\partial^{i-j}\left(a_{i}\left(0, \epsilon y+x_{\epsilon}\right) u\left(0, \epsilon y+x_{\epsilon}\right)\right)}{\partial^{i-j} t} \psi(y) d y .
$$


So there is a constant $D(\beta, \tau)>0$ such that

$$
\begin{aligned}
& -\sum_{i=1}^{k} \sum_{j=1}^{i} c_{\beta, j-1} \tau^{\beta-j+1} \epsilon^{N} \int_{\Omega_{1}} \frac{\partial^{i-j}\left(a_{i}\left(0, \epsilon y+x_{\epsilon}\right) u\left(0, \epsilon y+x_{\epsilon}\right)\right)}{\partial^{i-j} t} \psi(y) d y \\
& +D(\beta, \tau)\left(\sum_{|\alpha| \leq n} \epsilon^{N+\gamma \frac{r_{\alpha}^{\prime}}{r_{\alpha}}-|\alpha| r_{\alpha}^{\prime}}+\epsilon^{N+\gamma \frac{q^{\prime}}{q}}\right) \geq 0 .
\end{aligned}
$$

Now if $q<\gamma m_{\alpha} /|\alpha|$, for all $|\alpha| \leq n$, then, we have $N+\gamma \frac{r_{\alpha}^{\prime}}{r_{\alpha}}-|\alpha| r_{\alpha}^{\prime}>N$; so, if we divide (2.27)by $\epsilon^{N}$ and letting $\epsilon \rightarrow 0$ then from Fatou lemma we get:

$$
\begin{aligned}
& -\sum_{i=1}^{k} \sum_{j=1}^{i} c_{\beta, j-1} \tau^{\beta-j+1} \int_{\Omega_{1}} \frac{\partial^{i-j}\left(a_{i}(0,0) u(0,0)\right)}{\partial^{i-j} t} \psi(y) d y \\
& \geq 0
\end{aligned}
$$

But if one of the $\frac{\partial^{i-j}\left(a_{i}(0,0) u(0,0)\right)}{\partial^{i-j} t}$ be positive then we have a contradiction.

On the other hand, suppose that one of the initial data have a critical at zero and the other are positive. For example, suppose that for some $\delta>0$ there is an $\epsilon_{0}$ such that $|x|<\epsilon_{0}$ then $\frac{\partial^{i}\left(a_{i}(0, x) u(0, x)\right)}{\partial^{i} t}>|x|^{-\delta}$. So we get:

$$
-c_{\beta, \tau} \epsilon^{N-\delta} \int_{\Omega_{1}}|y+(0, \ldots, 0,2)|^{-\delta} \psi(y)+D(\beta, \tau)\left(\sum_{|\alpha| \leq n} \epsilon^{N+\gamma \frac{r_{\alpha}^{\prime}}{r_{\alpha}}-|\alpha| r_{\alpha}^{\prime}}+\epsilon^{N+\gamma \frac{q^{\prime}}{q}}\right) \geq 0 .
$$

Let $n^{\prime}=\min \left\{|\alpha| ; b_{\alpha}(t, x) \neq 0\right\}$.

Theorem 2.5. 1-Suppose $q<\gamma m_{\alpha} /|\alpha|$ and for some $1 \leq i \leq j \leq k$, $\frac{\partial^{i-j}\left(a_{i}(0, x) u(0, x)\right)}{\partial^{i-j} t}$ is continue and positive in origin. Then we have no nontrivial solution.

2-Suppose for some $1 \leq i \leq j \leq k, \frac{\partial^{i-j}\left(a_{i}(0, x) u(0, x)\right)}{\partial^{i-j} t}>|x|^{-\delta}$ and we have one of the following items

(a) $\delta>n$ and $\gamma<n^{\prime}$ or $\delta<n^{\prime}$ and $\delta>n$

(b) $\delta<\gamma$ and $n<\gamma$ or $\gamma<\delta$ and $\delta<n^{\prime}$

then we have no nontrivial solution.

Proof. 1 is proved. For 2; in each item $(a)$ or $(b)$ we have $N-\delta<\min \left(N+\gamma \frac{r_{\alpha}^{\prime}}{r_{\alpha}}-\right.$ $\left.|\alpha| r_{\alpha}^{\prime}, N+\gamma \frac{q^{\prime}}{q}\right)+$. Let $\epsilon \rightarrow 0$ in (2.29) then the left hand side will be negative which is a contradiction.

Corollary 2.6. 1-With the conditions of Theorem 2.4 we have no positive solution on $\mathbb{R}^{N}$ for the following problem:

$$
\sum_{|\alpha| \leq n} D^{\alpha}\left(b_{\alpha}(x) u^{m_{\alpha}}(x)\right) \geq c(x)|u(x)|^{q}
$$


2-With the conditions of part (1) Theorem (2.5) we have no positive continue solution on $\Omega-\{0\}$ (which $\Omega$ contains origin) for the following inequality:

$$
\sum_{|\alpha| \leq n} D^{\alpha}\left(b_{\alpha}(x) u^{m_{\alpha}}(x)\right) \geq|x|^{-\gamma}|u(x)|^{q} .
$$

Proof. In contrary, suppose that (2.30) have a solution. Let $u(t, x)=u(x)$. Then the problem $(2.1)$ for $F(u)=|u|^{q}$ have a positive solution on $\mathbb{R}^{N}$; which is a contradiction. The proof of 2 is similar.

REMARK 2.7. In (2.1) we can substitute $\sum_{i=1}^{k} \frac{\partial^{i}}{\partial t^{i}}\left(a_{i}(t, x) u(t, x)\right)$ by [4] $\sum_{i=1}^{k} \frac{\partial^{i}}{\partial t^{i}}\left(a_{i}(t, x) u^{o_{i}}(t, x)\right)$ where $0<o_{i}<q$ and in Theorem2.3 we can use the estimates similar to what we done in (2.7) and (2.14). Moreover, we must have some additional conditions.

REMARK 2.8. In the proof of Theorem (2.2) (a) we could choose any positive function in $C_{0}^{\infty}(\Omega)$ instead of $\phi$, by choosing large $\sigma$.

3. Laplacian type inequalities. In this section we consider the following problem

$$
\left\{\begin{aligned}
\sum_{i=1}^{k} \frac{\partial^{i}}{\partial t^{i}}\left(a_{i}(t, x) u(t, x)\right) & +\sum_{s=1}^{l} \Delta^{s}\left(b_{s}(t, x) u^{m}(t, x)\right), \\
& \geq c(t, x) F(u(t, x)) \\
\left.u^{m}(t, x)\right|_{\partial \Omega}=\left.\Delta\left(u^{m}(t, x)\right)\right|_{\partial \Omega}=\cdots=\left.\Delta^{l-1}\left(u^{m}(t, x)\right)\right|_{\partial \Omega}=0 & \\
u \geq 0 &
\end{aligned}\right.
$$

where $\left.\right|_{\partial \Omega}=0$ is in the mean of trace and $\Omega$ is a bounded smooth domain.

Here we shall show the global solution does not exist. Note that this inequality is a special case of the inequality (2.1), but here we can substitute $p-1>n+1$ with of $p>2$.

Assume that $a_{i}(t, x), b_{s}(t, x), c(t, x), A_{i}$, are as before and $b(t,.) \in C^{2 l-1}(\Omega)$. Let $B_{s}$ be the upper bound for $b_{s}(t, x)$ and $\vec{B}=\left(B_{1}, \ldots, B_{l}\right)$, and suppose $a_{i}(t, x) \geq 0$ In order to avoid ambiguty in this section we change the definition of the solution as follows.

Definition 3.1. By a solution $u$ of the problem (3.1) we mean a function $u \in C\left((0, T), C^{2 l-1}(\Omega)\right)$, which for any test function $\zeta$, which is given in the definition 2.1 , the function $u$ satisfies the folowing inequality:

$$
\begin{gathered}
\sum_{i=1}^{k} \sum_{j=1}^{i}(-1)^{j} \int_{\Omega} \frac{\partial^{i-j}\left(a_{i}(0, x) u(0, x)\right)}{\partial t^{i-j}} \frac{\partial^{j-1} \zeta(0, x)}{\partial t^{j-1}} d x+ \\
\sum_{j=1}^{k}(-1)^{j} \int_{0}^{T} \int_{\Omega} a_{j}(t, x) u(t, x) \frac{\partial^{j} \zeta(t, x)}{\partial t^{j}} d x d t+
\end{gathered}
$$




$$
\begin{gathered}
-\sum_{s=1}^{l} \int_{0}^{T} \int_{\Omega} \nabla \triangle^{s-1}\left(b_{s}(t, x) u^{m}(t, x)\right) \cdot \nabla(\zeta(t, x)) d x d t \\
\geq \int_{0}^{T} \int_{\Omega} c(t, x) F(u(t, x)) \zeta(t, x) d x d t
\end{gathered}
$$

TheOrem 3.2. Let $\phi, \lambda$ be as in the proof of Theorem 2.3, and $\psi$ be defined by:

$$
\psi:=\mu \phi,
$$

where

$$
\mu>\sum_{s=1}^{l} b_{s} \lambda^{s}
$$

$\left.a^{\prime}\right)$ Let $F(u)=u^{q}$. Then there is a positive number $M_{1}^{\prime}=M_{1}^{\prime}(\Omega, \tau, \vec{A}, \vec{B}, C)$ such that problem (3.1) has no nonnegative nontrivial global solution on $(0, \tau)$ when

$$
\sum_{i=1}^{k} \sum_{j=1}^{i} c_{\beta, j-1} \tau^{\beta-j+1} \int_{\Omega} \frac{\partial^{i-j}\left(a_{i}(0, x) u(0, x)\right)}{\partial^{i-j} t} \psi(x) d x>M_{1}^{\prime} .
$$

$\left.b^{\prime}\right)$ Let $F(u)=\left|\nabla u^{q}\right|^{p}$ and $p>2$. Then there is a positive number $M_{2}^{\prime}=$ $M_{2}^{\prime}(\Omega, \tau, \vec{A}, \vec{B}, C)$ such that (3.1) has no nonnegative nontrivial global solution on $(0, \tau)$ when:

$$
\sum_{i=1}^{k} \sum_{j=1}^{i} c_{\beta, j-1} \tau^{\beta-j+1} \int_{\Omega} \frac{\partial^{i-j}\left(a_{i}(0, x) u(0, x)\right)}{\partial^{i-j} t} \psi(x) d x>M_{2}^{\prime} .
$$

Proof. Now let as in the proof of Theorem 2.2

$$
\hat{\phi}(t):=\left\{\begin{array}{lr}
(\tau-t)^{\beta} & 0 \leq t \leq \tau, \\
0 & t>\tau
\end{array}\right.
$$

and $\beta-i q^{\prime}+1>1,1 \leq i \leq k$ and,

$$
\zeta(t, x):=\hat{\phi}(t) \psi(x) .
$$

By using the above $\zeta$ in the definition 3.1 and $u^{m}=\cdots=\Delta^{l-1} u^{m}=0$ on $\partial \Omega$ and concidering the Green's theorem, we get

$$
\begin{aligned}
& -\sum_{i=1}^{k} \sum_{j=1}^{i} \tau^{\beta-j+1} c_{\beta, j-1} \int_{\Omega} \frac{\partial^{i-j}\left(a_{i}(0, x) u(0, x)\right)}{\partial t^{i-j}} \psi(x) d x \\
& +\sum_{i=1}^{k} \int_{0}^{\tau} \int_{\Omega} c_{\beta, i}(\tau-t)^{\beta-i} a_{i}(t, x) u(t, x) \psi(x) d x d t \\
& +\sum_{s=1}^{l} \int_{0}^{\tau} \int_{\Omega}(\tau-t)^{\beta} b_{s}(t, x) u^{m}(t, x) \triangle^{s} \psi(x) d x d t \\
& \geq \int_{0}^{\tau} \int_{\Omega} c(t, x)(\tau-t)^{\beta} F(u(t, x)) \psi(x) d x d t .
\end{aligned}
$$


Case 1: part ( $\left.a^{\prime}\right)$. Similar to what we have done in the inequality (2.6) we can write:

$$
\begin{aligned}
& \int_{0}^{\tau} \int_{\Omega} c_{\beta, i}(\tau-t)^{\beta-i} a_{i}(t, x) u(t, x) \psi(x) d x d t \\
& \leq \int_{0}^{\tau} \int_{\Omega} K(\tau-t)^{\frac{\beta}{q}} \psi^{\frac{1}{q}}(x)|u(t, x)| K^{-1} A_{i} c_{\beta, i}(\tau-t)^{\frac{\beta}{q^{\prime}}-i} \psi^{\frac{1}{q^{\prime}}}(x) d x d t \\
& \leq \frac{K^{q}}{q} \int_{0}^{\tau} \int_{\Omega}(\tau-t)^{\beta} u^{q}(t, x) \psi(x) d x d t+\mu \frac{c_{\beta, i}^{q^{\prime}} A_{i}^{q^{\prime}}}{q^{\prime} K^{\frac{q^{\prime}}{q}}} \frac{\tau^{\beta-i q^{\prime}+1}}{\beta-i q^{\prime}+1}
\end{aligned}
$$

where $C(\psi)=\int_{\Omega} \psi(x) d x=\mu \int_{\Omega} \phi(x) d x=\mu$.

Again similar to inequality (2.7) we get:

$$
\begin{aligned}
& \int_{0}^{\tau} \int_{\Omega}(\tau-t)^{\beta} b_{s}(t, x) u^{m}(t, x) \psi(x) d x \\
& \leq \frac{K_{s}^{r}}{r} \int_{0}^{\tau} \int_{\Omega}(\tau-t)^{\beta}|u(t, x)|^{q} \psi(x)+\frac{B_{s}^{r^{\prime}} \tau^{\beta+1}}{r^{\prime} K_{s}^{r^{\prime}}(\beta+1)} \int_{\Omega} \frac{\left|\triangle^{s} \psi(x)\right|^{r^{\prime}}}{\psi^{\frac{r^{\prime}}{r}}} d x . \\
& \leq \frac{K_{s}^{r}}{r} \int_{0}^{\tau} \int_{\Omega}(\tau-t)^{\beta} u^{q}(t, x) \psi(x) d x+\frac{\lambda^{s r^{\prime}} \mu B_{s}^{r^{\prime}} \tau^{\beta+1}}{r^{\prime} K_{s}^{r^{\prime}}(\beta+1)}
\end{aligned}
$$

where $r=\frac{q}{m}$ and

$$
\int_{\Omega} \frac{\left|\Delta^{s} \psi(x)\right|^{r^{\prime}}}{\psi(x)^{\frac{r^{\prime}}{r}}} d x=\lambda^{-s r^{\prime}} \int_{\Omega} \frac{\psi^{r^{\prime}}(x)}{\psi^{\frac{r^{\prime}}{r}}} d x=\lambda^{-s r^{\prime}} \int \psi(x) d x=\lambda^{-s r^{\prime}} \mu .
$$

By using (3.5) and (3.6) in (3.4) let $F(u)=u^{q}$ we get:

$$
\begin{aligned}
& -\sum_{i=1}^{k} \sum_{j=1}^{i} \tau^{\beta-j+1} c_{\beta, j-1} \int_{\Omega} \frac{\partial^{i-j}\left(a_{i}(0, x) u(0, x)\right)}{\partial t^{i-j}} \psi(x) d x \\
& +\sum_{i=1}^{k} \mu \frac{c_{\beta, i}^{q^{\prime}} A_{i}^{q^{\prime}}}{q^{\prime} K^{q^{\prime}}} \frac{\tau^{\beta-i q^{\prime}+1}}{\beta-i q^{\prime}+1}+\sum_{s=1}^{l} \frac{\lambda^{-s r^{\prime}} \mu B_{s}^{r^{\prime}} \tau^{\beta+1}}{r^{\prime} K_{s}^{r^{\prime}}(\beta+1)} \\
& \geq\left(C-\frac{k K^{q}}{q}-\sum_{s=1}^{l} \frac{K_{s}^{r}}{r}\right) \int_{0}^{\tau} \int_{\Omega}(\tau-t)^{\beta} u^{q}(t, x) \psi(x) d x d t .
\end{aligned}
$$

Now choose $K>0, K_{s}>0$ small, $1 \leq s \leq l$, and let

$$
M_{1}^{\prime}=\sum_{i=1}^{k} \mu \frac{c_{\beta, i}^{q^{\prime}} A_{i}^{q^{\prime}}}{q^{\prime} K^{q^{\prime}}} \frac{\tau^{\beta-i q^{\prime}+1}}{\beta-i q^{\prime}+1}+\sum_{s=1}^{l} \frac{\lambda^{-s r^{\prime}} \mu B_{s}^{r^{\prime}} \tau^{\beta+1}}{r^{\prime} K_{s}^{r^{\prime}}(\beta+1)} .
$$

प 
Case 2: part $\left(b^{\prime}\right)$. Similar to (2.11) we can write:

$$
\begin{aligned}
& \int_{0}^{\tau} \int_{\Omega} c_{\beta, i}(\tau-t)^{\beta-i} a_{i}(t, x) u(t, x) \psi(x) d x d t \\
& \leq \frac{K^{q} \lambda}{q p} \int_{0}^{\tau} \int_{\Omega}(\tau-t)^{\beta}\left|\nabla u^{q}(t, x)\right|^{p} \psi(x) d x+\frac{K^{q} \lambda \tau^{\beta+1}}{q p^{\prime}(\beta+1)} D_{2}(\phi) \\
& +\frac{c_{\beta, i}^{q^{\prime}} A_{i}^{q^{\prime}}}{q^{\prime} K^{q^{\prime}}} \frac{\tau^{\beta-i q^{\prime}+1}}{\beta-i q^{\prime}+1} D_{1}(\phi) \\
& \leq \frac{K^{q} \lambda}{q p} \int_{0}^{\tau} \int_{\Omega}(\tau-t)^{\beta}\left|\nabla u^{q}(t, x)\right|^{p} \psi(x) d x+\frac{K^{q} \lambda \tau^{\beta+1}}{q p^{\prime}(\beta+1)} D_{2}(\phi) \\
& +\frac{c_{\beta, i} A_{i}^{q^{\prime}}}{q^{\prime} K^{q^{\prime}}} \frac{\tau^{\beta-i q^{\prime}+1}}{\beta-i q^{\prime}+1} \mu^{q^{\prime}},
\end{aligned}
$$

where $K>0$ is a real number and $D_{1}(\phi)=\int_{\Omega} \frac{\psi^{q^{\prime}}(x)}{\phi(x)^{\frac{q^{\prime}}{q}}} d x=\mu^{q^{\prime}}$. Also from $p>2$ by Lemma (2.3) we get:

$$
D_{2}(\phi)=\int_{\Omega} \frac{|\nabla \phi(x)|^{p^{\prime}}}{\psi(x)^{\frac{p^{\prime}}{p}}}=\frac{1}{\mu^{\frac{p^{\prime}}{p}}} \int_{\Omega} \frac{|\nabla \phi(x)|^{p^{\prime}}}{\phi(x)^{\frac{p^{\prime}}{p}}}<\infty .
$$

Similar to $(2.14)$

$$
\begin{aligned}
& \int_{0}^{\tau} \int_{\Omega}(\tau-t)^{\beta} b_{s}(t, x) u^{m}(t, x) \Delta^{s} \psi(x) d x \\
& \leq \frac{K_{s}^{r} \lambda}{r p} \int_{0}^{\tau} \int_{\Omega}(\tau-t)^{\beta}\left|\nabla u^{q}(t, x)\right|^{p} \psi(x) d x+\frac{K_{s}^{r} \lambda \tau^{\beta+1}}{r p^{\prime}(\beta+1)} D_{2}(\phi) \\
& +\frac{B_{s}^{r^{\prime}} \tau^{\beta+1}}{r^{\prime} K_{s}^{r^{\prime}}(\beta+1)} D_{3}(\phi, s) \\
& \leq \frac{K_{s}^{r} \lambda}{r p} \int_{0}^{\tau} \int_{\Omega}(\tau-t)^{\beta}\left|\nabla u^{q}(t, x)\right|^{p} \psi(x) d x \\
& +\frac{K_{s}^{r} \lambda \tau^{\beta+1}}{r p^{\prime}(\beta+1)} D_{2}(\phi)+\frac{B_{s}^{r^{\prime}} \tau^{\beta+1}}{r^{\prime} K_{s}^{r^{\prime}}(\beta+1)} \lambda^{-s p^{\prime}} \mu^{p^{\prime}},
\end{aligned}
$$

where $D_{3}(\phi, s)=\int_{\Omega} \frac{\left|\Delta^{s} \psi(x)\right|^{p^{\prime}}}{\phi(x)^{\frac{p^{\prime}}{p}}} d x=\lambda^{-s p^{\prime}} \mu^{p^{\prime}}$.

By using (3.7) and (3.8) in (3.4) we get

$$
\begin{aligned}
& -\sum_{i=1}^{k} \sum_{j=1}^{i} \tau^{\beta-j+1} c_{\beta, j-1} \int_{0}^{\tau} \int_{\Omega} \frac{\partial^{i-j}\left(a_{i}(0, x) u(0, x)\right)}{\partial t^{i-j}} \psi(x) d x \\
& +\sum_{i=1}^{k} \frac{c_{\beta}^{q^{\prime}} A_{i}^{q^{\prime}}}{q^{\prime} K^{q^{\prime}}} \frac{\tau^{\beta-i q^{\prime}+1}}{\beta-i q^{\prime}+1} \mu^{q^{\prime}}+\frac{k K^{q} \lambda \tau^{\beta+1}}{q p^{\prime}(\beta+1)} D_{2}(\phi) \\
& +\tau^{\beta+1} \sum_{s=1}^{l}\left(\frac{K_{s}^{r} \lambda}{r p^{\prime}(\beta+1)} D_{2}(\phi)+\frac{B_{s}^{r^{\prime}} \lambda^{-s p^{\prime}} \mu^{p^{\prime}}}{r^{\prime} K_{s}^{r^{\prime}}(\beta+1)}\right) \\
& \geq\left(C-\frac{k K^{q} \lambda}{p q}-\sum_{s=1}^{l} \frac{K_{s}^{r} \lambda}{r p}\right) \int_{0}^{\tau} \int_{\Omega}(\tau-t)^{\beta}\left|\nabla u^{q}(t, x)\right|^{p} \psi(x) d x d t .
\end{aligned}
$$


Now we choose $K$ and $K_{s}, 1 \leq s \leq k$ small and we let

$$
M_{2}^{\prime}=\sum_{i=1}^{k} \mu \frac{c_{\beta, i}^{q^{\prime}} A_{i}^{q^{\prime}}}{q^{\prime} K^{q^{\prime}}} \frac{\tau^{\beta-i q^{\prime}+1}}{\beta-i q^{\prime}+1}+\sum_{s=1}^{l} \frac{\lambda^{-s r^{\prime}} \mu B_{s}^{r^{\prime}} \tau^{\beta+1}}{r^{\prime} K_{s}^{r^{\prime}}(\beta+1)} .
$$

Acknowledgment. The authors acknowledge the support of Sharif University of Technology grant during this research.

\section{REFERENCES}

[1] D. Andreucci, Degenerate parabolic equations with initial data measuers, Transaction of the American Mathematical Socity, 349 (1997), pp. 3911-3923.

[2] H. Bellout, S. Benachour, E.S. Titi, Finite-time singularity versus global regularity for hyper-viscous Hamilton-Jacobi-like equations, Nonlinearity, 16 (2003), pp. 1967-1989.

[3] J.M. BALL, Remarks on blow-up and nonexistence theorems for nonlinear evolution equations, Quart. J. Math. Oxford, 28 (1977), pp. 473-486.

[4] H. FujitA, On some nonexistence and nonuniqueness theorems for nonlinear parabolic equations, Proc. Symp. Pure Math., XVIII, Nonlinear Functional Analysis, Amer. Math. Soc., 28 (1970), pp. 105-113.

[5] V. GeORGiev AND G. Todorova, Existence of solution of the wave equation with nonlinear damping and source terms, C.R. Acad. Sci. Paris, Serie I, 314 (1992), pp. 205-209.

[6] R.T. Glassey, Blow-up theorems for nonlinear wave equations, Math. Z., 132 (1973), pp. 183-203.

[7] S. Kaplan, On the growth of solutions of quasilinear parabolic equations, Comm. Pure. Appl. Math., 16 (1963), pp. 327-343.

[8] G.G. LAPTEv, Some nonexistence results for higher-order evolution inequality in cone-like domains, Electronic Research Announcements of the American Mathematical Society, 7 (2001), pp. 87-93.

[9] G.G. LAPTEV, Nonexistence results for higher-order evolution partial differential inequalities, Proc. Amer. Math. Soc., 131:2 (2003), pp. 415-423.

[10] H. Levine, Instability and nonexistence of global solutions to nonlinear wave equations of the form $P u_{t t}=A u+F(u)$, Trans. Amer. Math. Soc., 192 (1974), pp. 1-21.

[11] H.Levine, Some additional remarks on the nonexistence of global solutions to nonlinear wave equations, S.I.A.M. J. Math. Anal., 5 (1974), pp. 541-553.

[12] E. Mitidieri and S. I. Pohozaev, A priori estimates and blow-up of solutions to nonlinear partial differential equations and inequalities, Proc. Steklov Inst. Math., 234 (2001).

[13] S.I. Pohozaev And A. Tesei, Blow-up of nonnegative solutions to quasilinear parabolic inequalities, Atti Accad. Naz. Lincei Rend. Cl. Sci. Fis. Mat., 11 (2000), pp. 99-109.

[14] S.I. Pohozaev And A. TeSeI, Instantaneous blow-up of solutions to a class of hyperbolic inequalities, Electronic Journal of Differential Equations, (2002), pp. 155-165.

[15] $\mathrm{PH}$. Souplet, Gradient blow-up for multidimensional nonlinear parabolic equations with general boundary conditions, Diff. Integral Equations, 15 (2002), pp. 237-256.

[16] Рh. Souplet, Proprietes globales de quelques equations d'evolution du second ordre non lineaires, These, Universite Pierre et Marie Curie, Paris, (1993), pp. 116-127.

[17] M. Tsutsumi, On solutions of semilinear differential equations, Math. Japonicae, 17 (1972), pp. $173-193$. 
H. ASSA AND M. HESAARAKI 